Communications in Applied Geometry.

Volume 1, Number 1 (2011) pp. 31-36

(C) Research India Publications

https://dx.doi.org/10.37622/CAG/1.1.2011.31-36

\title{
A Symmetry of Optic Phenomena
}

\author{
Euichi Miztani \\ 3-63, Yamabushi, Nishi-Imajuku, Ama, Aichi, Japan
}

\begin{abstract}
Generally, when we see an object, it is projected up side down through the crystalline lens on the retina. However, we can see it normally because the brain recovers the upset picture. Thus, our neurosurgical projection system through eyes and brain seems to be somewhat complicate. However, there is an interesting symmetry on the projection system. The aim of this paper is to simplify it by mathematics with a geometric model for the optic phenomenon. We furthermore discuss to replace it by two fundamental rules of arithmetics by addition and subtraction.
\end{abstract}

Keywords: retinal symmetry, clock model, duodecimal system, fiber optics.

\section{Introduction}

In recent years, patients affected with retinal disease tend to increase. For example, AMD(Age-related Macular Degeneration) and Diabetic Retinopathy are major ones. It will be therefore significant to detect and identify the exact location of the affected area on retina. However, it is complicated for the above reason. For example, when an ophthalmologist find an affected part like macular hole through ophthalmo-scope, the patient's retina with macular hole(; black spot) is as shown in A of Figure 1. However, the patient would see it as if $A^{\prime}$ of Figure 1(; if s/he could recognized the macular hole.) The relation of two sights A and A' is symmetric, and both of them are true. However, the macular hole(; white spot) is actually located at where it is done a one-eighty; they are rotational symmetric each other. 

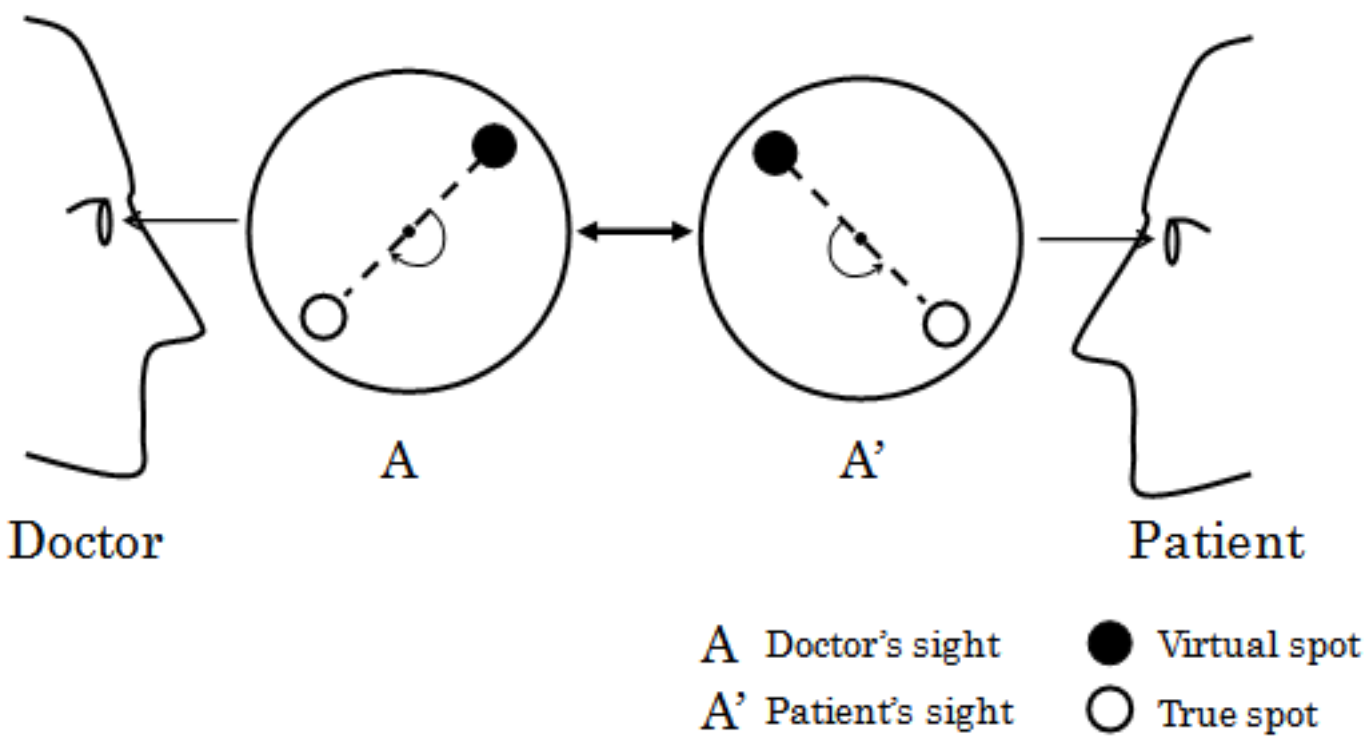

Figure 1

If explaining the phenomenon concretely, Let us draw the projection system by a graph with refracting rays of light through lenses as shown in Figure 2 and 3. It shows how our human brains project an object into our minds.

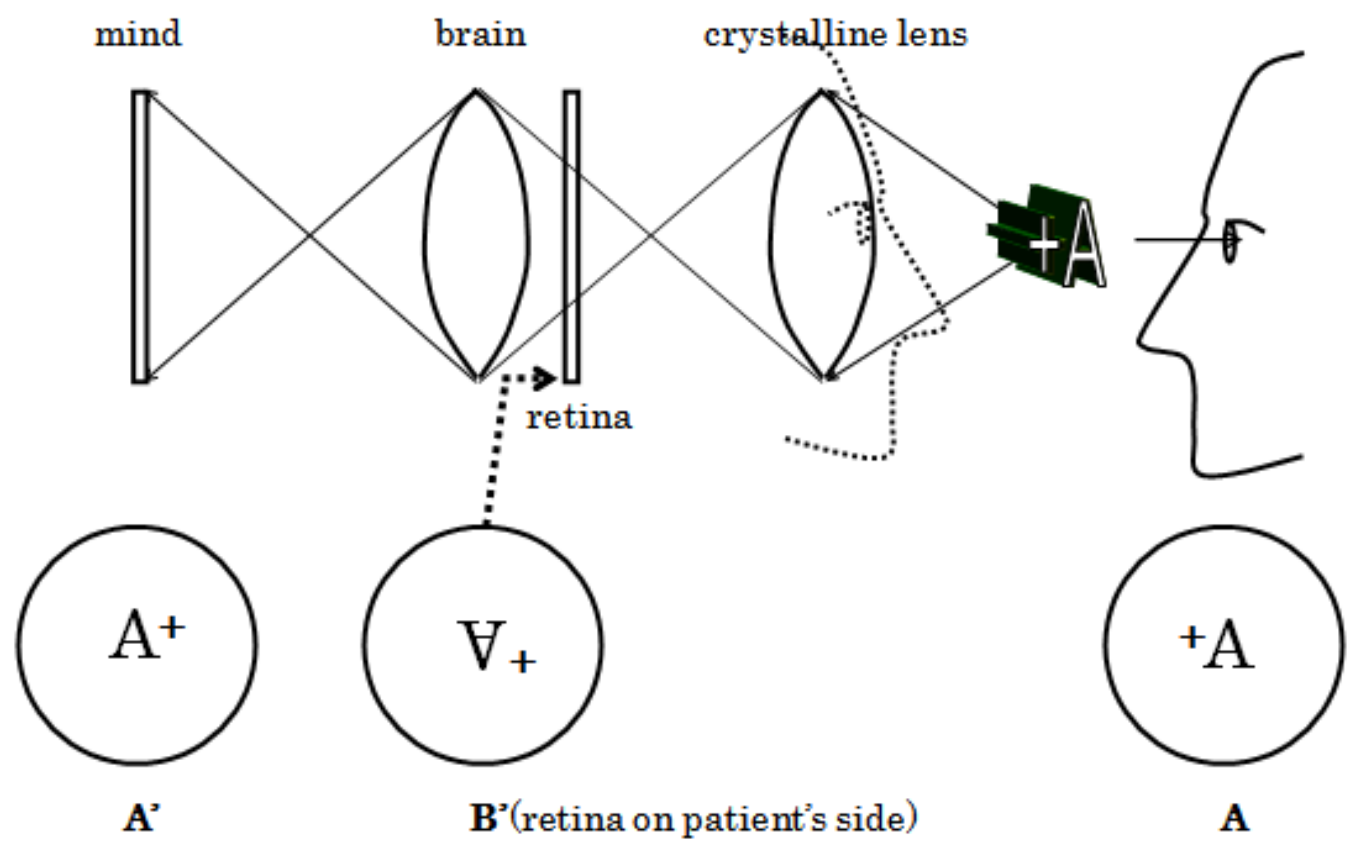

Figure 2 


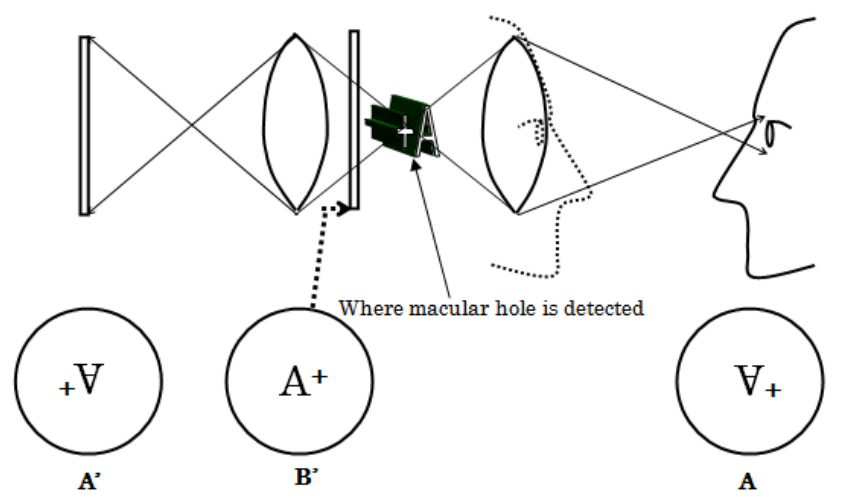

Figure 3

\section{Analysis by Graphs}

To understand the phenomenon analytically, first of all, let us introduce a simple model with clock boards. These boards are mainly used as calibrations. The model is as shown in Figure 4; all boards are transparent and these two sets of lined up three clocks are all the same but they are set opposite to each other. Additionally, the boards $\mathrm{A}^{\prime}$ are reversed to others. Now, using spot instead of $\mathrm{A}^{+}$and explaining the location by time number on the clock, let us think of the analysis by graph. According to the virtual and true spot on the left-hand model of Figure 4 by A-B-A' order, the location is at 1 o'clock(; virtual), 7 o'clock(; true), and 11 o'clock(; virtual). Thus, we can simply identify the location of macular hole on retina with the clock model.

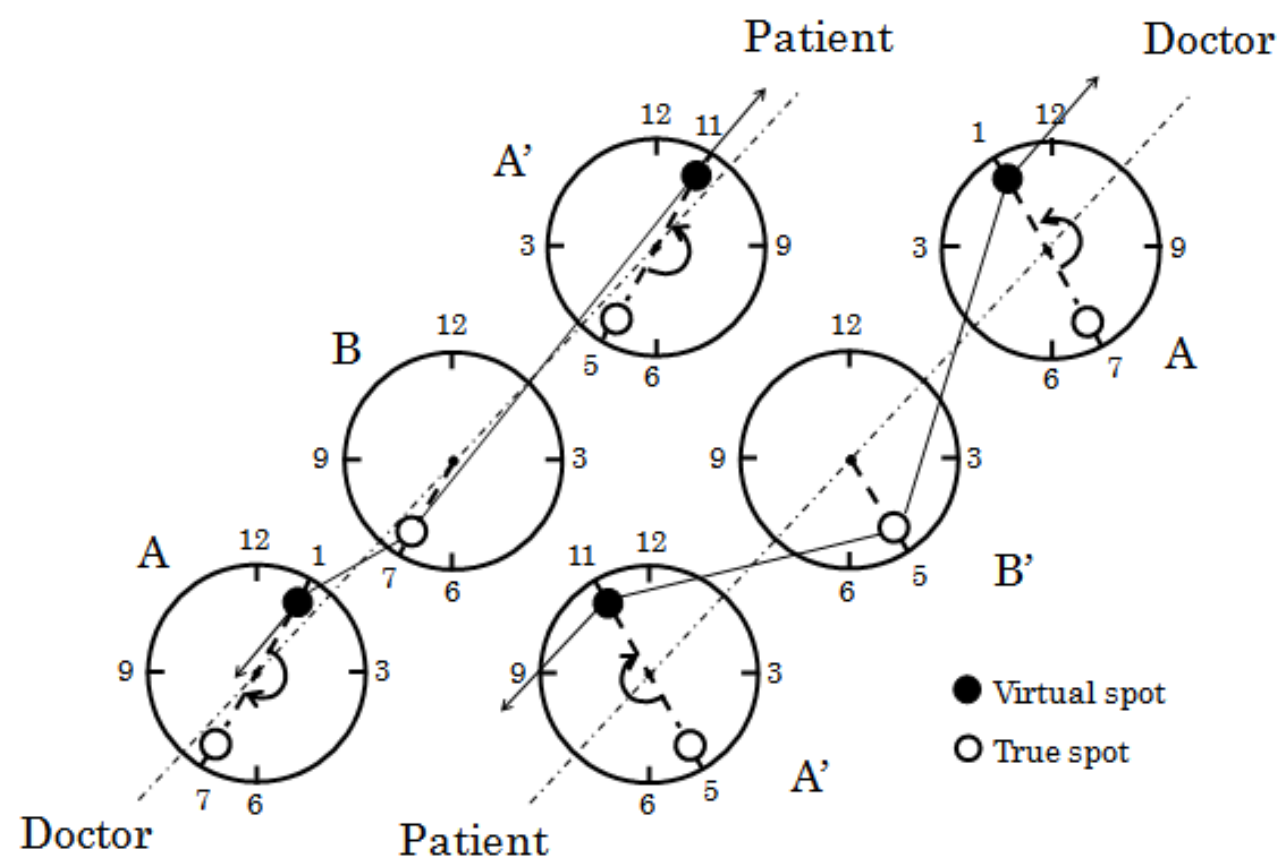

Figure 4 


\section{Conversion to Algebra}

Furthermore, we can make sure the location simpler by simple arithmetic. Let us introduce a diagram as shown in Fig.5. It shows two numbers on the left and right on the clock, and every total of 5(or 7) pairs is twelve. Another important fact is half-turn advance equals to 6 hours advance on the clock.

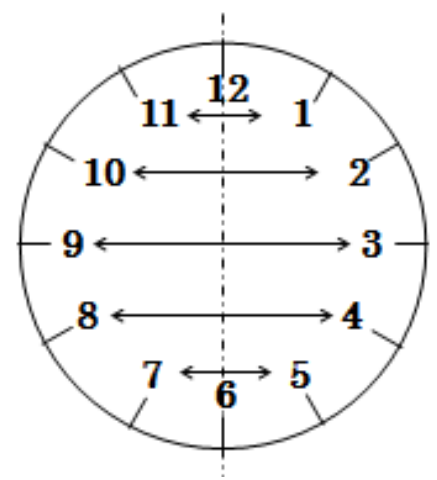

\begin{tabular}{|c|c|c|}
\hline $\mathbf{L}$ & & $\mathbf{R}$ \\
\hline ( 12 & + & 0) \\
\hline 11 & + & 1 \\
\hline 10 & + & 2 \\
\hline 9 & + & 3 \\
\hline 8 & + & 4 \\
\hline 7 & + & 5 \\
\hline (6 & + & 6) \\
\hline
\end{tabular}

\section{Total(L+R)}

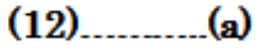

12

12

12

$12 \ldots . . . . . .(e)$

$12 \ldots(\mathbf{f})$

(12)

\section{Figure 5}

Now, let us calculate the locations of the spot by these laws with referring to the geometric clock system above mentioned. If a patient see the spot at 11 o'clock in his or her sight, doctor can identify the location by arithmetic of (b) on the diagram, the virtual location from doctor's sight $D v s$ is

$$
\text { Dvs }=12-11=1 \text { (o'clock) (; virtual spot on A) }
$$

The true location on the retina from doctor's sight $D t s$ is

$$
D t s=D v s+6=1+6=7 \text { (o'clock) (; true spot on B) }
$$

If doctor explain where the true spot is in patient's sight Pts , the equation is

$$
\text { Pts }=11+6=17 .
$$

Since the time(o'clock) is ruled by the duodecimal system,

$$
\text { Pts }=17 \bmod 12 \text { = } 5 \text { (o'clock) (; true spot on A') }
$$

They correspond to the left hand model of Figure 4. Thus, all locations are understandable by combination of two rules of operation above. Again,

1. In the relation of virtual spot on retina between doctor and patient, either is located at 12-x o'clock if another is at x o'clock.

2. The true spot is at where advanced 6 hours from the virtual spot

\section{Application to the Fiber Optics}

The arithmetic method with the clock model will work for the fiber optics. Generally, 
when we look at an object through single convex lens, it is projected up side down. The case by two lenses is discussed above. Now, if using more than two lenses, it will be further difficult to identify how object is seen. However, the arithmetic method actually facilitates identification of cases more than two lenses as well. First of all, let us confirm of how an object is seen by refractions through three lenses. It is as shown in Figure 6.

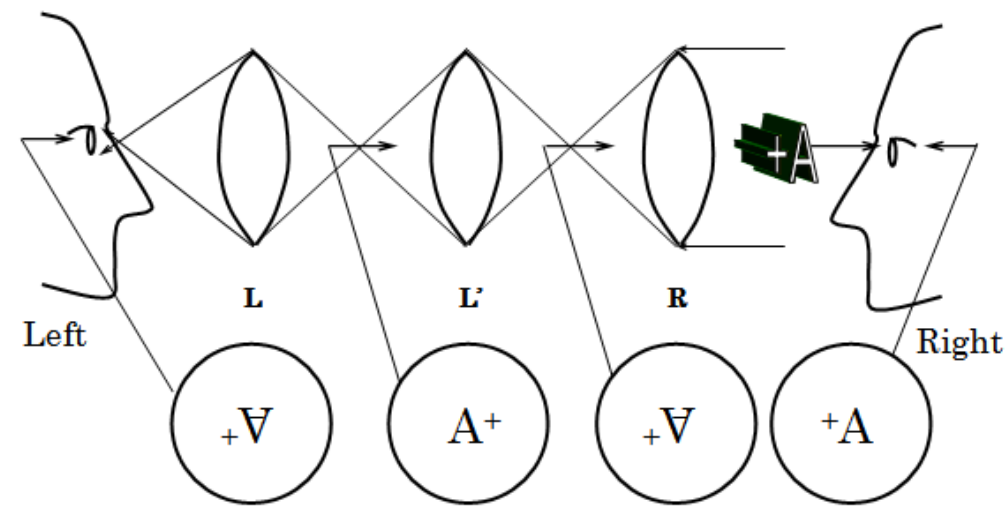

Figure 6

To understand it analytically, let us introduce the clock model again. It is as shown in Figure 7. Since L' and R are reversed, so that the time order is anticlockwise from viewpoint on the left.

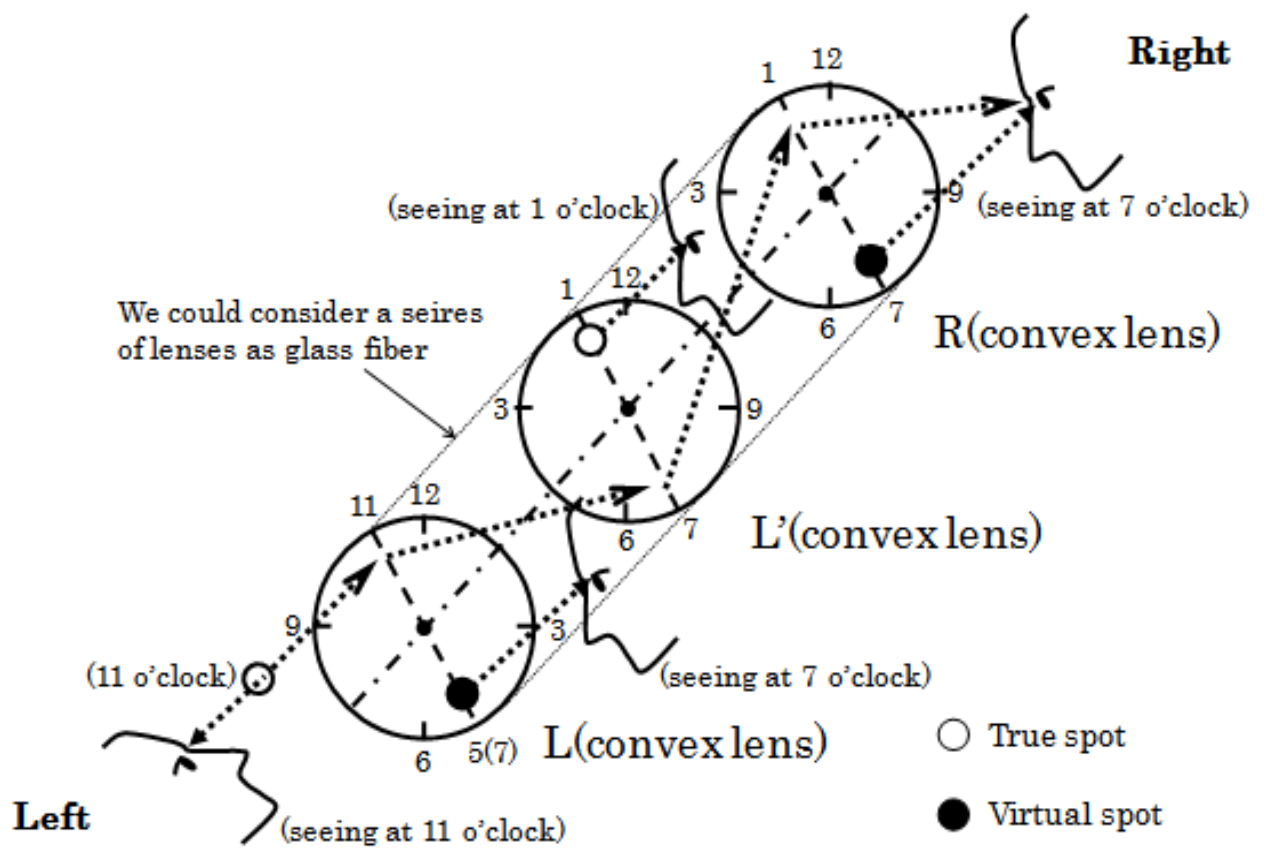

Figure 7 
For example, assume that a person at $\mathrm{L}$ from left side saw a true spot at 11 o'clock, the other one at L' from right side did it as shown in figure 7. Then, we can calculate the location by (b) of the diagram of Figure 5 as follows;

$$
\text { L to } \mathrm{L}^{\prime}=12-11=1 \text { (o’clock). }
$$

Seeing it at $\mathrm{R}$ from right side, the equation is

$$
\mathrm{L} \text { to } \mathrm{R}=12-11+6=7 \text { (o'clock). }
$$

Therefore, we can make sure of all the locations by two arithmetic laws below. If an object is at $t$ o'clock, the location through lenses is identified as

$$
\left\{\begin{array}{lc}
\left\{L_{t}, R\right\}^{N}=12-t & (N=2 n) \\
\left\{L_{t}, R\right\}^{N}=12-t+6(\bmod 12) & (N=2 n-1)
\end{array} .\right.
$$

$N$ denotes the number of lenses or number of refractions in the glass fiber.

\section{Acknowledgement}

The retinal or optical symmetry was discovered from dialogs with ophthalmologists in the process of treatments of my left eye's detached retina. I acknowledge the ophthalmologists of Nagoya University Hospital. 